\title{
Performance of biodegradable microcapsules of poly(butylene succinate), poly(butylene succinate-co-adipate) and poly(butylene terephthalate-co-adipate) as drug encapsulation systems
}

\author{
Cornelia Theresa Brunner ${ }^{\mathrm{a}, \mathrm{b}, \mathrm{c}}$, Erkan Türker Baran ${ }^{\mathrm{a}, \mathrm{b}, *}$, Elisabete Duarte Pinho ${ }^{\mathrm{a}, \mathrm{b}}$, \\ Rui Luís Reis ${ }^{\mathrm{a}, \mathrm{b}}$, Nuno Meleiro Neves ${ }^{\mathrm{a}, \mathrm{b}}$ \\ a 3B's Research Group - Biomaterials, Biodegradables and Biomimetics, University of Minho, Headquarters of the European Institute \\ of Excellence on Tissue Engineering and Regenerative Medicine, AvePark, S. Cláudio do Barco, 4806-909 Taipas, Guimarães, Portugal \\ ${ }^{\mathrm{b}}$ IBB - Institute for Biotechnology and Bioengineering, PT Government Associated Laboratory, Guimarães, Portugal \\ c Department of Engineering Sciences, Martin-Luther University Halle-Wittenberg, D-06099 Halle/S, Germany
}

\section{A R T I C L E I N F O}

\section{Article history:}

Received 21 September 2010

Received in revised form 14 January 2011

Accepted 1 February 2011

Available online 3 March 2011

\section{Keywords:}

Controlled drug release

Microcapsule

Poly(butylene succinate)

Poly(butylene succinate-co-adipate)

Poly(butylene terephthalate-co-adipate)

\begin{abstract}
A B S T R A C T
Poly(butylene succinate) (PBSu), poly(butylene succinate-co-adipate) (PBSA) and poly(butylene terephthalate-co-adipate) (PBTA) microcapsules were prepared by the double emulsion/solvent evaporation method. The effect of polymer and poly(vinyl alcohol) (PVA) concentration on the microcapsule morphologies, drug encapsulation efficiency (EE) and drug loading (DL) of bovine serum albumin (BSA) and all-trans retinoic acid (atRA) were all investigated. As a result, the sizes of PBSu, PBSA and PBTA microcapsules were increased significantly by varying polymer concentrations from 6 to $9 \%$. atRA was encapsulated into the microcapsules with an high level of approximately 95\% EE. The highest EE and DL of BSA were observed at $1 \%$ polymer concentration in values of 60 and 37\%, respectively. $4 \%$ PVA was found as the optimum concentration and resulted in 75\% EE and 14\% DL of BSA. The BSA release from the capsules of PBSA was the longest, with $10 \%$ release in the first day and a steady release of $17 \%$ until the end of day 28. The release of atRA from PBSu microcapsules showed a zero-order profile for 2 weeks, keeping a steady release rate during 4 weeks with a 9\% cumulative release. Similarly, the PBSA microcapsules showed a prolonged and a steady release of atRA during 6 weeks with $12 \%$ release. In the case of PBTA microcapsules, after a burst release of $10 \%$ in the first day, showed a parabolic release profile of atRA during 42 days, releasing $36 \%$ of atRA.
\end{abstract}

(c) 2011 Elsevier B.V. All rights reserved.

\section{Introduction}

The delivery of protein drugs and bioactive agents which promote cell growth and guide cell differentiation is crucial for tissue engineering [1]. Proteins and bioactive agents need a prolonged release to show their maximum activity. Therefore, they benefit if protected from the immune system and the degrading environment of body fluids and from being washed away from the target site. In addition, the release of bioactive agents like growth factors must be long enough to obtain maximum biological effect [2]. Delivery systems incorporating growth factors, and mainly biodegradable

\footnotetext{
* Corresponding author at: 3B's Research Group - Biomaterials, Biodegradables and Biomimetics, University of Minho, Headquarters of the European Institute of Excellence on Tissue Engineering and Regenerative Medicine, AvePark, S. Cláudio do Barco, 4806-909 Taipas, Guimarães, Portugal. Tel.: +351 253510 916; fax: +351253510909.

E-mail addresses: corneliabrunner@gmail.com (C.T. Brunner), turker.baran@dep.uminho.pt (E.T. Baran), epinho@dep.uminho.pt (E.D. Pinho), rgreis@dep.uminho.pt (R.L. Reis), nuno@dep.uminho.pt (N.M. Neves)
}

particles, could offer distinctive advantages. The ability to regulate the release rate while protecting the loaded protein during all the stages of tissue regrowth, enables having consistent signals to be delivered locally which is very relevant for developing new tissue engineering strategies $[3,4]$.

The bioactive substance may be released by a diffusion mechanism or by degradation of the carrier. However, the kinetics of dug release is far more complex in the degradation mediated process due to the eventual drug interaction with the carrier polymer and its degradation products [5]. Also, the emulsifying agents used in its production can affect the release kinetics significantly. Both, the dosage and the half-life of the drug can be controlled by the macromolecular chemistry and the degradation rate of the polymer [6]. Based on the polymeric systems used and on the drug release profiles, the release models may be distinctly categorized as diffusion-controlled, swelling-controlled, or erosion controlled systems [7]. There is a synergistic effect between diffusion and erosion that condition the release kinetics. In particular, the rate of erosion, the effective diffusion coefficient of the drug molecule in the wet polymer, and the average pore dimensions, are found to 
<smiles>CCOC(=O)CCC(=O)OCCCC(C)(C)C</smiles><smiles>CCCCCCOC(=O)CCC(=O)OCCCCC(=O)OCCCC(=O)OC(C)C</smiles><smiles>CC(C)CCCCOC(=O)CCCCC(=O)OC(C)C</smiles>

Fig. 1. Chemical formulas of PBSu, PBSA and PBTA polyesters

be critical parameters affecting the kinetics of release. [8]. In addition, effects of water-in-oil-in-water process parameters can play significant roles in the release of hydrophilic macromolecules from microspheres by the surface porosity and internal morphology [9].

Poly(butylene succinate) (PBSu), poly(butylene succinate-coadipate) (PBSA), and poly(butylene terephthalate-co-adipate) (PBTA) (Fig. 1) are all synthetic polyesters produced by polycondensation of butanediol and different dicarboxylic acids: succinic acid for PBSu, succinic/adipic acid for PBSA. The copolyesters displayed a faster degradation rate than that of PBSu [10]. Poly(butylene succinate) and its copolymers are relatively recent and they have not been studied in detail as biomaterials for application in drug delivery as compared with other synthetic biodegradable polyesters such as poly(l-lactide) (PLLA), poly(lactide-co-glycolide) (PLGA), poly ( $\varepsilon$-caprolactone) (PCL). Recently, the in vitro evaluation of $\mathrm{PBSu}$ as a biomaterial has showed promising results in terms of cytotoxicity as well as proliferation and differentiation of osteoblasts and mesenchymal stem cells seeded on scaffolds produced using those materials [11-14].

In this study, we investigated the potential of PBSu and its copolymers as a microcapsule drug delivery system, indented for prolonged release of bioactive agents. For that purpose, the hydrophilic bovine serum albumin (BSA, 66 kDa) and all-trans retinoic acid (atRA, $300.4 \mathrm{~g} / \mathrm{mol}$ ) as a hydrophobic drug were encapsulated into PBSu and copolymer microcapsules. The double emulsion/solvent evaporation technique was used to prepare the microcapsules, which is considered suitable for solvent sensitive bioactive agents and for protein drugs. In addition, the atRA is an effective agent as it has roles in the differentiation of bone, epithelia, nervous system, immune system and skin [15]. The low solubility of this hydrophobic of character of atRA in aqueous solutions is a major barrier to its application and therefore, the microcapsules proposed in this study may constitute a solution overall those limitations.

\section{Materials and methods}

\subsection{Materials}

PBSu (the average molecular weight $\left(M_{\mathrm{w}}\right) 89000$, the numberaverage molecular weight $\left.\left(M_{\mathrm{n}}\right) 34000\right)$ and PBSA $\left(M_{\mathrm{w}} 184000\right.$, $M_{\mathrm{n}} 97$ 000) were obtained from Showa Highpolymer Ltd. (Tokyo, Japan). PBTA ( $\left.M_{\mathrm{w}} 78000, M_{\mathrm{n}} 43000\right)$ was obtained from Eastman Chemical Co. (Kingsport, Tennessee, USA). Poly vinyl alcohol (PVA) ( $M_{\mathrm{W}}$ 30000-70000), dichloro methane, all-trans retinoic acid and bovine serum albumin were all obtained from Sigma Aldrich Chemical Co. (St Luis, USA).

All the chemicals used herein were analytical grade.

\subsection{Contact angle measurements}

Contact angle measurements were performed to determine the hydrophilicity of the polyesters. For this test, it is necessary to have a flat surface, thus membranes were prepared by casting of a solution of the polyesters in dichloromethane. A drop of $3 \mu$ l of HPLC grade $\mathrm{H}_{2} \mathrm{O}$ was placed at the surface of the polyester membrane and the initial contact angle was measured in the Contact Angle System OCA-dataphysics.

\subsection{Preparation of PBSu, PBSA and PBTA microcapsules}

A schematic depiction of the preparation procedure of the polyester microcapsules can be seen in Fig. 2. The preparation conditions including type of polymer, polymer concentration, type of encapsulated drug, concentration of emulsifier in the second aqueous phase as well as in the evaporation solution are all summarized in Table 1.

The microcapsules were prepared by a modified double emulsion/solvent evaporation technique as described previously [16]. Briefly, the polymer pellets were dissolved in $1.8 \mathrm{ml}$ of dichloromethane. For the encapsulation of atRA, the drug ( $2 \mathrm{mg}$ ) was dissolved in a polymer solution and $0.3 \mathrm{ml}$ of distilled water was used as first aqueous phase $\left(w_{1}\right)$ while the albumin $(18 \mathrm{mg})$ was dissolved in $0.3 \mathrm{ml}$ phosphate buffered saline solution (PBS, $\mathrm{pH}$ 7.4). After adding $w_{1}$, the single emulsion was formed by vortex mixing for $20 \mathrm{~s}$. Immediately after, the second aqueous phase $\left(w_{2}\right)$, $6 \mathrm{ml}$ aqueous PVA solution as emulsifier, was added and the double emulsion was formed by subsequent vortex mixing for another $20 \mathrm{~s}$. The resultant emulsion was poured into a $40 \mathrm{ml}$ aqueous PVA solution $(0.5 \%, \mathrm{w} / \mathrm{v})$ and stirred on a magnetic stirrer for $1.5 \mathrm{~h}$ at 200 revolutions per minute (rpm) to evaporate the solvent and harden the microcapsules.

To collect the microcapsules, they were filtered through a mixed cellulose ester membrane (Whatman, Dassel-Germany) with a pore size of $0.45 \mu \mathrm{m}$. Then, they were rinsed three times with distilled water and finally transferred into closed and light protected containers (for atRA) and stored at $4{ }^{\circ} \mathrm{C}$ until further use.

In order to calculate encapsulation efficiency and drug loading, the protein content of the supernatants (not encapsulated) was measured by the dye binding (Bradford) method, as this protein determination method does not interfere with PVA in supernatants [17]. Briefly, the Bradford solution was prepared by dissolving $50 \mathrm{mg}$ Brilliant Blue G (Sigma Chemical Co., St Louis, USA) in $50 \mathrm{ml}$ ethanol (95\%) and $100 \mathrm{ml}$ phosphoric acid (85\%) solution and completed to $1 \mathrm{~L}$ with distilled water. Both the protein sample and the standard BSA solutions $(0.5 \mathrm{ml})$ were mixed with the $5 \mathrm{ml}$ of filtered Bradford reagent solution and incubated for $5 \mathrm{~min}$ at room temperature. Subsequently, the colour intensity developed was measured at $595 \mathrm{~nm}$ by using UV-vis spectrophotometer (Shimadzu 2100). The percent $(0.1 \%)$ extinction coefficient of BSA standard concentrations (between $5 \times 10^{-3}$ and $2.5 \times 10^{-2} \mathrm{mg} / \mathrm{ml}$ ) was detected as $12.4(\mathrm{mg} / \mathrm{ml})^{-1} \mathrm{~cm}^{-1}$.

atRA in filtrates and in washing solutions was determined by UV-vis absorbance spectrophotometry at $312 \mathrm{~nm}$. The extinction coefficient $(0.1 \%)$ from atRA standard concentration

\section{Table 1}

Preparation conditions of PBSu, PBSA and PBTA microcapsules for investigation of influence of emulsifier and polymer concentration on drug (BSA) encapsulation efficiency and drug loading.

\begin{tabular}{lll}
\hline Polyesters & $\begin{array}{l}\text { Polyester concentration } \\
(\%, \mathrm{w} / \mathrm{v})\end{array}$ & $\begin{array}{l}\text { PVA concentration in } \\
w_{2}(\%, \mathrm{w} / \mathrm{v})\end{array}$ \\
\hline PBSu, PBSA, PBTA & $1 ; 3 ; 6 ; 9$ & 2 \\
& 5 & $0.5 ; 1 ; 2 ; 4 ; 6$ \\
\hline
\end{tabular}




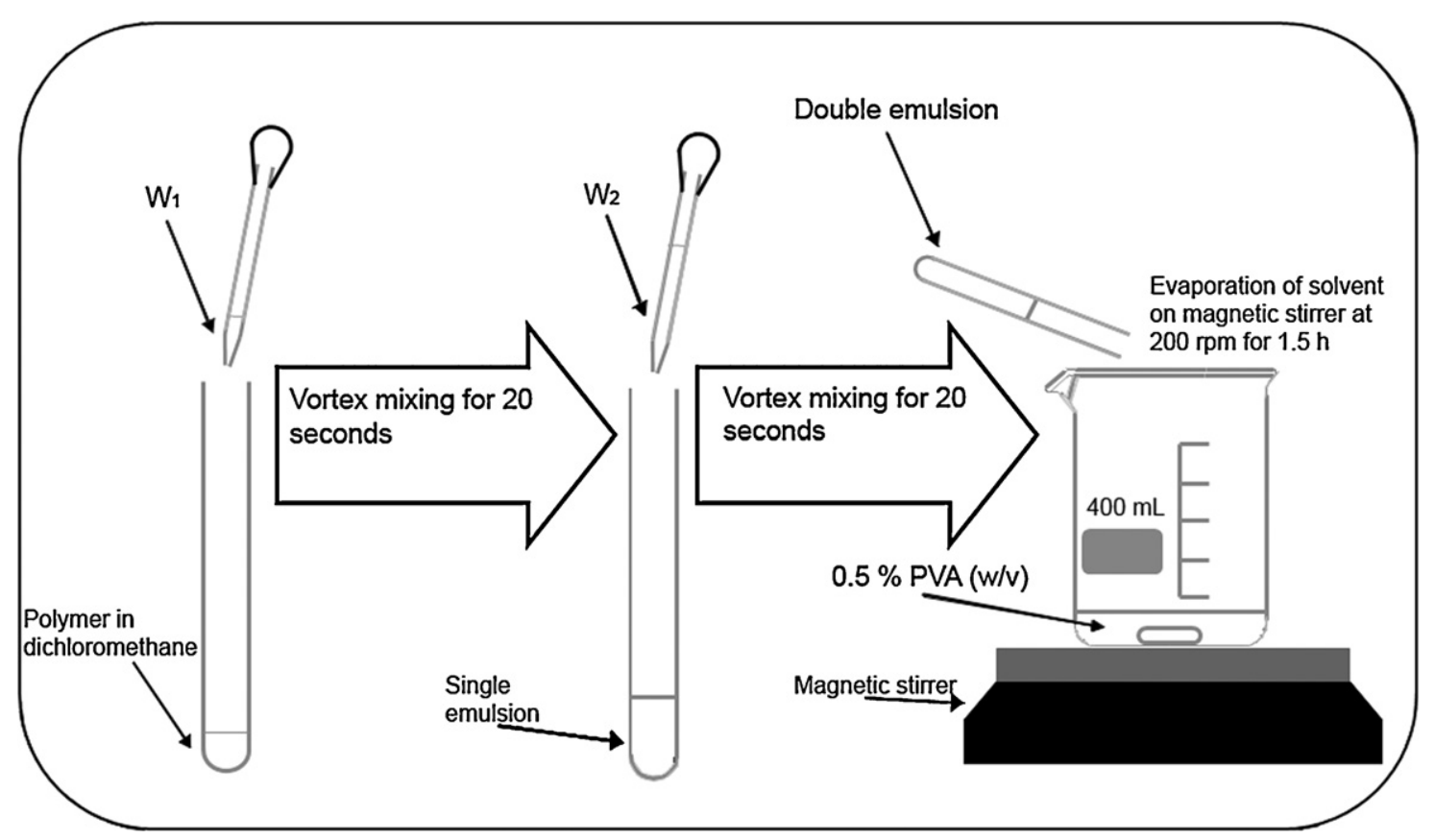

Fig. 2. Schematic representation of the sequence of steps followed on the preparation of microcapsules by double emulsion/solvent evaporation technique.

(between $3.3 \times 10^{-3}$ and $2 \times 10^{-2} \mathrm{mg} / \mathrm{ml}$ ) readings was found as $24.9(\mathrm{mg} / \mathrm{ml})^{-1}$.

The encapsulation efficiency (EE) was determined according to the following equation:

$\mathrm{EE}=\left(\frac{m_{\mathrm{d}}}{m_{\mathrm{l}}}\right) \times 100$

where EE is the encapsulation efficiency; $m_{\mathrm{d}}$ is the weight of encapsulated drug; $m_{1}$ is the total weight of drug used during preparation.

The drug loading (DL) was determined according to the equation below:

$\mathrm{DL}=\left(\frac{m_{\mathrm{d}}}{m_{\mathrm{d}}+m_{\mathrm{p}}}\right) \times 100$

where DL is the drug loading; $m_{\mathrm{d}}$ is the weight of encapsulated drug; $m_{\mathrm{p}}$ is the weight of polymer.

\subsection{Microcapsule characterization}

\subsubsection{Scanning electron microscopy}

For scanning electron microscopy (SEM) analysis of the samples, aqueous suspensions of washed microcapsules were dried at room temperature on carbon coated SEM-stubs and sputter coated with gold. For the morphology characterization, the polyester microcapsules were examined using a SEM S360 equipment by Leica Cambridge. The obtained SEM micrographs were examined for the characterization of the morphology of the various microcapsules.

\subsubsection{Size determination of microcapsules}

Dilute aqueous suspensions of washed microcapsules were placed on microscope slides and covered by a cover slide. Those specimens were further analyzed by transmission light microscopy (TLM) and representative micrographs were obtained by using a digital camera. The obtained TLM micrographs were processed with the Image image analysis software (National Institute of Health, USA, version $1.38 \times$ ), for the determination of the average size of the polyester microcapsules. The high resolution images were transformed into 8-bit images and a micrograph of scale of calibration slide, obtained at the same microscope magnification, was used to calibrate the software. Then, the diameters of the microcapsules were characterized manually by drawing a line across the diameter of the microcapsules. Aggregates of particles and irregular-shape particles were excluded from the analysis, even if they were rare. At least 100 microcapsules were measured for each preparation condition by analyzing random visual fields of the micrographs from different batches of samples.

\subsection{In vitro drug release studies}

The drug release kinetics was studied on microcapsules produced at a polymer and PVA concentration of 5 and $2 \mathrm{wt} . \%$, respectively. The microcapsules ( $450 \mathrm{mg}$ dry weight) were immersed into $40 \mathrm{ml}$ PBS solution in a 50-ml-Falcon tube and incubated in a water bath at $37^{\circ} \mathrm{C}$ and $100 \mathrm{rpm}$ shaking rate. For each study, a triplicate of the release batches was tested. The control preparations were also used in the release studies for providing blank solutions in photometric measurements. The control batches were made as described above microcapsule preparation procedures except that no drug was added into respective phases during encapsulation process. In order to measure the protein release, the media contents were allowed to settle down and the drawn aliquots at low volume $(1 \mathrm{ml})$ were replaced with fresh release medium as this method can provide enough sink conditions for highly water soluble molecules like BSA as explained by D'Souza SS and DeLuca [18]. For the BSA release study, a PBS solution containing $0.02 \%$ sodium azide was used to inhibit microbial growth. At defined time points, $1 \mathrm{ml}$-aliquots were extracted from the solution and being replaced by fresh PBS solution. The albumin content of aliquots was determined by the Lowry assay [19].

For measuring atRA in vitro, the whole release medium was exchanged with fresh PBS solution periodically to prevent saturation of drug in the release medium and to make a sink condition as explained by Choi et al. [20]. For atRA release, the light protected Falcon tubes were utilized during all the study. At predetermined time points, shaking was stopped for 10 min to allow for the microcapsules to settle down. Then, the whole release medium was decanted and filtered to recover remaining microcapsules. Microcapsules on filter membrane were washed into the respective 
Table 2

The contact angle of air and glass-contact surface of polymer.

\begin{tabular}{lrc}
\hline & Air contact $\left(^{\circ}\right)$ & Glass contact $\left(^{\circ}\right)$ \\
\hline PBSu & $108.22 \pm 0.14$ & $111.81 \pm 0.32$ \\
PBSA & $99.26 \pm 0.32$ & $108.47 \pm 0.73$ \\
PBTA & $84.65 \pm 0.41$ & $100.80 \pm 0.96$ \\
\hline
\end{tabular}

Falcon tube by rinsing with the fresh PBS solution and the volume was completed to $40 \mathrm{ml}$ to continue the release study. Then, the filtrate volume was measured and atRA content was determined by UV-vis absorbance at $312 \mathrm{~nm}$.

\subsection{Statistical analysis}

Unless otherwise indicated, the quantitative results were acquired from at least triplicate samples. The data were expressed as means and error bars show standard deviation (S.D.). The statistical analysis for size determination was carried out by using unpaired Student's $t$-test using Origin 6.0 software program (Microcal Software Inc., Northampton, MA, USA), and a value of ${ }^{*} P<0.05$ was considered statistically significant.

\section{Results and discussions}

\subsection{Contact angle study}

Contact angle measurements were performed to ascertain the wetting nature of each dry polyester film. The polymer microstructure and hydrophilic/hydrophobic interactions also play an important role in determining drug distribution profiles and release characteristics. The difference in water contact angle values of air and glass contact films can be attributed to the differences in surface roughness. The smooth surfaces of the membrane casted in contact with the glass result consistently in higher values of the contact angle (Table 2). The PBSu and PBSA polyesters showed a hydrophobic character with air water contact angle of $108.22^{\circ} \pm 0.14^{\circ}$ and $99.26^{\circ} \pm 0.32^{\circ}$, respectively (Table 2 ). PBTA was the only polyester tested exhibiting a contact angle lower than $90^{\circ}$, the value obtained being $84.65 \pm 0.41$ (Table 2 ) and demonstrated some relatively hydrophilic nature. The hydrophilicity of the polyester may facilitate the permeation of water into the microcapsule structure and, consequently, it is believed to facilitate the drug diffusion rate. For the release study it may be expected that the release rate can be relatively faster in the case of PBTA and slower in PBSu and PBTA microcapsules when smaller molecules are embedded at the capsule wall.

\subsection{Characterization microcapsules}

\subsubsection{Effect of polymer concentration on size and morphology}

The SEM photographs of microcapsules produced with various polymer concentrations are shown in Fig. 3. Both PBSA and PBTA microcapsules show very smooth surface and spherical shape at $1 \%$ polymer concentration. In the case of the PBSu microcapsules however, the microcapsules have a spherical shape with a rough surface and open pore structure. The rough surface morphology and open pore structures were also typical the typical morphology of PBSu microcapsules at higher polymer concentrations. The microcapsules prepared with 3\% PBSA and PBTA are also spherical in shape and are characterized mostly by a smooth surface. The microcapsules prepared with 6\% PBSA were spherical in shape and occasionally showed polygonal shape with a rough surface. At that concentration, the PBTA microcapsules were irregular in shape with rough surface, although small spherical microcapsules were also visible. The microcapsules prepared with 9\% PBSA were the roughest and shown some open pore structure with multiple particles inside the hollow capsules. At the highest polymer concentration, the PBTA microcapsules are characterized by a cage-like open pore structure.

The influence of polymer concentration on the average size of the microcapsules is summarized in Table 3. In general, the sizes of the microcapsules were quite variable for all preparations. That may reflect inhomogeneous mixing by the vortexing. The average size of the microcapsules depends considerably on the polymer concentration. The microcapsule size increased directly with the polymer concentration, reaching a maximum at $9 \%$ (either $P<0.05$ or $P<0.01$ ). The microcapsules were approximately $11.6,6.9$ and $8.1 \mu \mathrm{m}$ in diameter at $1 \%$ concentrations for the various polymers studied PBSu, PBSA and PBTA, respectively. The average size of the microcapsules increased slightly with the increase of the polymer concentration between 1 and 6\%. At 9\% concentration, however,

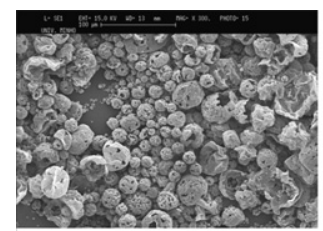

PBSu $1 \%$

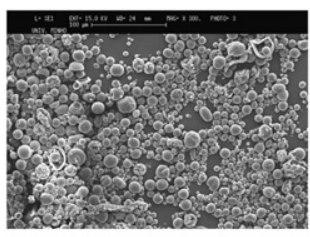

PBSA $1 \%$

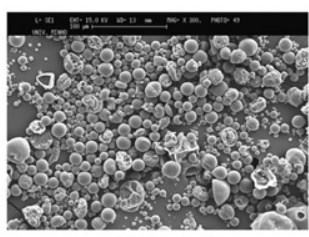

PBTA $1 \%$

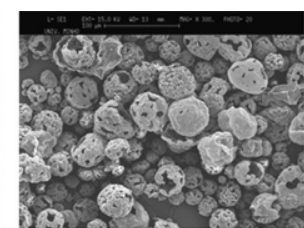

PBSu $3 \%$

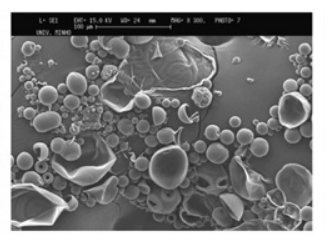

PBSA $3 \%$

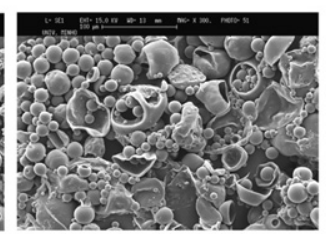

PBTA $3 \%$

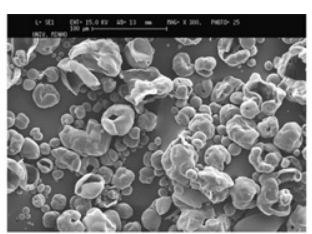

PBSu $6 \%$

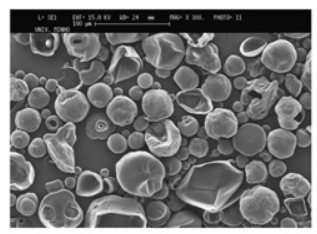

PBSA $6 \%$

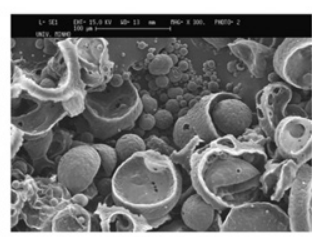

PBTA $6 \%$

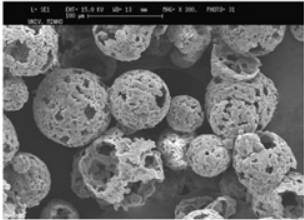

PBSu $9 \%$

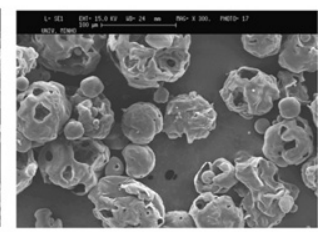

PBSA $9 \%$

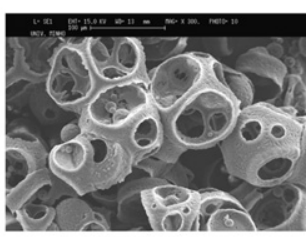

PBTA $9 \%$

Fig. 3. SEM of the microcapsules obtained using different polymer concentrations (magnification $300 \times$ ). 
Table 3

The average size of microcapsules which were prepared with the various polymer concentrations.

\begin{tabular}{|c|c|c|c|c|}
\hline \multirow[t]{2}{*}{ Type of polyester } & \multicolumn{4}{|c|}{ Size of microcapsules $[\mu \mathrm{m}]$ according polyester concentration } \\
\hline & $1 \%$ & $3 \%$ & $6 \%$ & $9 \%$ \\
\hline PBSu & $11.6 \pm 3.6$ & $17.9 \pm 3.1$ & $11.5 \pm 2.4$ & $70.2 \pm 3.4^{*}$ \\
\hline PBSA & $6.9 \pm 0.8$ & $10.2 \pm 2.1$ & $20.0 \pm 3.5^{*}$ & $72.8 \pm 3.4^{* *}$ \\
\hline PBTA & $8.1 \pm 2.0$ & $10.6 \pm 2.1$ & $13.6 \pm 2.1$ & $64.4 \pm 1.2^{* *}$ \\
\hline
\end{tabular}

$\pm:$ Standard deviation (100 readings). ${ }^{*}$ Differences are statistically significant when compared with $1 \%$ polymer concentration (unpaired Student's $t$-test, ${ }^{*} P<0.05$ or ${ }^{* *} P<0.01$ ).

the average microcapsule size increased dramatically when compared to the size observed at $6 \%$ for PBSu, PBSA and PBTA (Table 3 ). The porous morphology of the microcapsules was dominant at that polymer concentration. The average pore size was $14.5,18.0$ and $35.0 \mu \mathrm{m}$ for PBSu, PBSA and PBTA, respectively (data not shown in the tables).

The complex structures are apparent from SEM microphotographs of the microcapsules obtained when using higher polymer concentrations. Those results can be explained by the tendency to have coalescence of several microcapsules into a single structure, eventually including smaller capsules inside the larger structures. In addition, as the polymer solution becomes more viscous by increased polymer concentration, more energy is necessary for breaking the surface energy to form the droplets. This fact may increase the population of microcapsules with larger size since the mechanical energy was kept constant for all experiments. Similar increase of size and size distribution with increased polymer concentrations was already previously reported $[16,21,22]$. The size of the microcapsules, prepared by both $\mathrm{w} / \mathrm{o} / \mathrm{w}$ was determined to be highly affected by the intrinsic viscosity of the polymer in an exponential order [23]. Similarly, the size PLGA microsphere was shown to be increased with higher intrinsic viscosity of polymer solution [24,25].

In our studies, the open pore microcapsules and honeycomb structured surfaces were both observed in SEM micrographs for higher polymer concentrations, especially at $9 \%$. Those porous microcapsules might have interesting applications in tissue engineering to be used either as cell or drug carrier, since those pores are sufficiently large to enable cell infiltration. For example, Kim et al. reported that the highly porous PLGA microspheres with the surface average pore size (aps) at $20 \mu \mathrm{m}$ was found to enable the cell infiltration and seeding [26]. Likewise, the cell carriers with the surface pores larger than a cell size (i.e. $10 \mu \mathrm{m}$ ), such as the microporous cellulose beads [27], alginate beads [28], ceramic capsules (aps: 20-100 $\mu \mathrm{m}$ ) [29] and chitosan microspheres (aps: 35-60 $\mu \mathrm{m}$ )
[30], were proposed as suitable cell carriers for increasing cell attachment, proliferation, spreading and protection of cells from the shear stress.

In multiple emulsions, different morphologies are generated due to the enhanced probability of the presence of the aqueousphase microdroplets inside the emulsion droplets. During the solvent elimination stage, these microdroplets generally coalesce under the pressure of the precipitating polymer. Depending on the polymer concentration and the emulsification energies, the final microparticles will be a mixture of honeycomb, capsule or solid structures [31]. In a recent study, the retardation of indomethacin release kinetics by PCL coating has indicated that the porous morphology can be attributed for fast release kinetics of unmodified microparticles [32].

\subsubsection{Effect of PVA concentration size and morphology}

The concentration of emulsifier, PVA, had profound effects over the surface morphologies and average size of the microcapsules. For PBSu, spherical and rough-walled microcapsules were observed at all concentrations except for 1\% PVA, where they were smoother (Fig. 4). Large, rougher microcapsules were dominating the PBSA microcapsule population at $0.5 \%$ PVA concentration. At $1 \%$ and higher concentrations of PVA, on the other hand, smaller and smooth-walled PBSA microcapsules were obtained. Large, honey comb-shaped microcapsules mixed with smooth and rough-walled spherical microcapsules of PBTA were obtained at 0.5 and 1\% PVA. The obtained PBTA microcapsules were smoother and predominantly spherical between 2 and 6\% of PVA.

The size variation of microcapsules was very significant with high PVA concentrations $(P<0.05$ or $P<0.01)$. Large microcapsules, in average, were detected at $0.5 \% \mathrm{PVA}(28.2,39.2$ and $26.8 \mu \mathrm{m}$ diameter for, PBSu, PBSA and PBTA, respectively (Table 4). The average size of microcapsules dropped to $17.1 \mu \mathrm{m}$ in the case of PBSu, while a slight increase was observed for PBSA and PBTA microcapsules at $1 \%$ PVA concentration. The microcapsules of PBSA and PBTA pre-

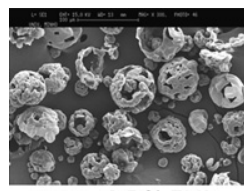

PBSu, $0.5 \%$ PVA

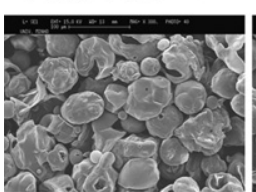

PBSA, $0.5 \%$ PVA

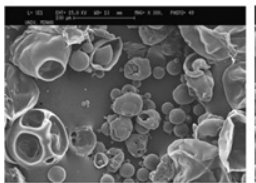

PBTA, $0.5 \%$ PVA

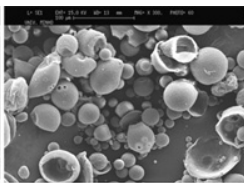

PBSu, $1 \%$ PVA

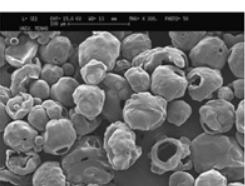

PBSA, $1 \%$ PVA

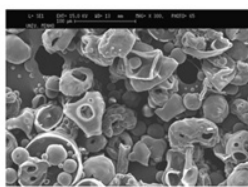

PBTA, $1 \%$ PVA

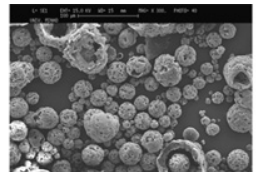

PBSu, 2 \% PVA

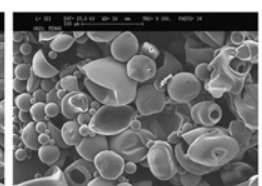

PBSA, 2 \% PVA

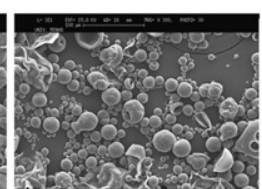

PBTA, $2 \%$ PVA

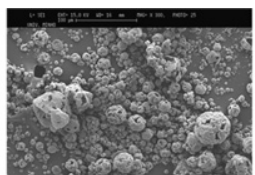

PBSu, 4 \% PVA

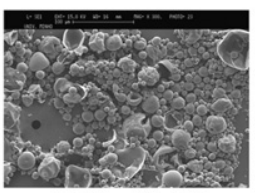

PBSA, 4 \% PVA

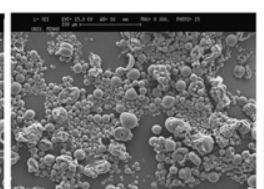

PBTA, $4 \%$ PVA

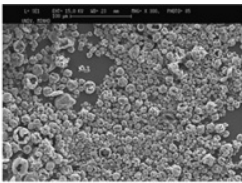

PBSu, $6 \%$ PVA

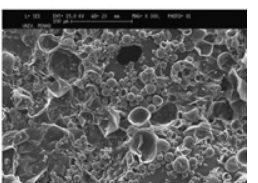

PBSA, $6 \%$ PVA

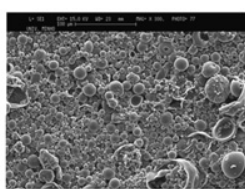

PBTA, $6 \%$ PVA

Fig. 4. SEM of the microcapsules obtained using different emulsifier (PVA) concentrations (magnification 300×). 
Table 4

The average size of microcapsules which were prepared by various PVA concentrations.

\begin{tabular}{|c|c|c|c|c|c|}
\hline \multirow[t]{2}{*}{ Type of polyester } & \multicolumn{5}{|c|}{ Size of microcapsules $[\mu \mathrm{m}]$ according PVA concentration in $w_{2}$} \\
\hline & $0.5 \%$ & $1 \%$ & $2 \%$ & $4 \%$ & $6 \%$ \\
\hline PBSu & $28.2 \pm 2.2$ & $17.1 \pm 0.8^{*}$ & $18.0 \pm 2.8$ & $12.0 \pm 0.5^{* *}$ & $6.5 \pm 1.4^{* *}$ \\
\hline PBSA & $39.2 \pm 2.2$ & $44.6 \pm 2.1^{*}$ & $21.3 \pm 4.7^{*}$ & $10.6 \pm 1.2^{* *}$ & $7.9 \pm 0.9^{* *}$ \\
\hline PBTA & $26.8 \pm 1.2$ & $37.9 \pm 0.2^{* *}$ & $12.2 \pm 0.3^{* *}$ & $5.4 \pm 1.0^{* *}$ & $7.1 \pm 1.8^{* *}$ \\
\hline
\end{tabular}

\pm : Standard deviation (100 reading). ${ }^{*}$ Differences are statistically significant when compared with $0.5 \%$ PVA concentration (unpaired Student's $t$-test, ${ }^{*} P<0.05$ or ${ }^{* *} P<0.01$ ).

pared at 2\% PVA showed an abrupt drop in size to 21.3 and $12.2 \mu \mathrm{m}$, respectively. At $4 \%$ PVA, further significant $(P<0.01)$ reductions in microcapsule sizes were detected $(12.0,10.6$ and $5.4 \mu \mathrm{m}$, respectively). Except for PBSu which showed a reduction from 12.0 to $6.5 \mu \mathrm{m}$, the average size of microcapsules of PBSA and PBTA did not changed significantly from 4 to $6 \%$ PVA.

Increasing the PVA concentration provides conditions to obtain smaller emulsion droplets resulting in the formation of smaller microcapsules. Thus, PBSu, PBSA and PBTA microcapsules sizes were observed to change accordingly. Similarly, a decrease of the size of PLGA nanoparticles and poly(hydroxybutyrate-covalerate) (PHBV) nanocapsules were already shown to result from the increase of PVA concentration in the emulsification medium $[33,34,16]$. It has also recently been shown that the PVA concentration in aqueous phases can change the particle morphology considerably, such as the aspect ratio of spheroidal microparticles of PLGA and poly(hydroxybutyrate) microparticles in a non-linear way $[35,36]$.

\subsection{Influence of preparation conditions on EE and DL}

In this study, the optimization of EE and DL was performed for a model protein, since hydrophilic compounds can easily diffuse into an external aqueous phase during double emulsion preparation and precipitation of the polymer [37]. On the other hand, the EE of atRA in microcapsules of all utilized polyesters is very high: 94.0, 95.4 and $95.8 \%$ for PBSu, PBSA and PBTA microcapsules, respectively. These high encapsulation efficiencies can be explained by the poor water solubility of atRA and easier solubility in hydrophobic polymer solutions. The SEM images of atRA-encapsulated microcapsules showed occasional crystallites at the surface of microcapsules wall (Fig. 5a). The SEM micrographs also show a hollow structure (Fig. 5b), proving that the drug was partitioned within the organic polymer phase during the microcapsule preparation. Consequently, the dispersion of atRA within the thin microcapsule wall can provide an efficient strategy for facilitating solubility and extending half-life of the drug in the body fluids by providing both a protection and controlled release mediated by the large surface area [38-41].

\subsubsection{Influence of polymer concentration}

The influence of the concentration of the polyester solution during microcapsule preparation on the encapsulation efficiency and drug loading of BSA are both shown in Fig. 6a and b, respectively. It was found that the EE is relatively higher (ca. 60\%) at the lowest polymer concentration (1\%). EE was not strongly affected by the variation in polymer concentration between 3 and $9 \%$, and the difference between the various copolymers at each concentration was not statistically significant. Those results show the efficiency of the use of polyesters in the encapsulation of proteins at relatively low polymer concentrations. DL, on the other hand, decreases profoundly with increasing polyester concentration, as it was expected from predictions based in the respective equation in which DL is inversely proportional to the polyester concentration. At $1 \%$ polymer concentration, the DL of the three polymers was nearly 37\%. DL dropped abruptly for 3, 6 and 9\% polymer concentrations, giving DL values ranging from 5 to $15 \%$ (Fig. 6b). Qualitatively, the behavior of the three polymers was very similar, without significant changes between the various copolymers.

\subsubsection{Influence of PVA concentration}

PVA was used as emulsifier with concentration ranges between 0.5 and $6 \%$ in the second water phase $\left(w_{2}\right)$ and showed having a strong effect on EE and DL. The PVA concentration at $0.5 \%(\mathrm{w} / \mathrm{w})$ produced around $75 \% \mathrm{EE}$ of BSA for PBSu, PBSA and PBTA polyester microcapsules (Fig. 6c). At 1\% PVA concentration, however, both PBSu and PBSA were at 50\% while PBTA was at $62 \%$. Keeping similar trends, the EE values of PBSu, PBSA and PBTA were observed to be close to $60 \%$ when it was used a $2 \%$ PVA concentration. At $4 \%$ PVA concentration it was observed the highest EE with over $75 \% \mathrm{EE}$ for the tested polyester microcapsules. When compared with the previous PVA concentrations, the $6 \%$ condition showed a significant drop in EE for PBSu, PBSA and PBTA, with values of 63,70 and $55 \%$, respectively. When compared the $E E$ of the three polymers for the same PVA concentration, the 6\% PVA group was the most sensitive to the polymer type.

As a function of EE, the DL profiles of BSA for the three polyester microcapsules were larger at $4 \%$ and then at $0.5 \%$ PVA, with values of 12 and $14 \%$, respectively (Fig. 6d). At $1 \%$ PVA concentration, where the DL values were the smallest, the DL of PBSu and PBSA were approximately $9 \%$ while for PBTA was approximately $11 \%$. At the
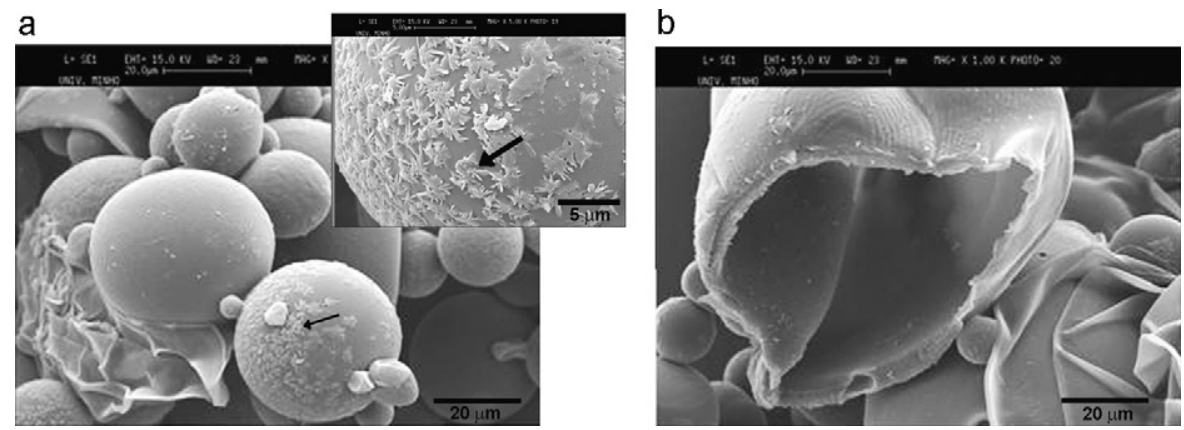

Fig. 5. Scanning electron microscopy image of a group of atRA encapsulated PBSu microcapsules (a) and cross section of a microcapsule (b). 

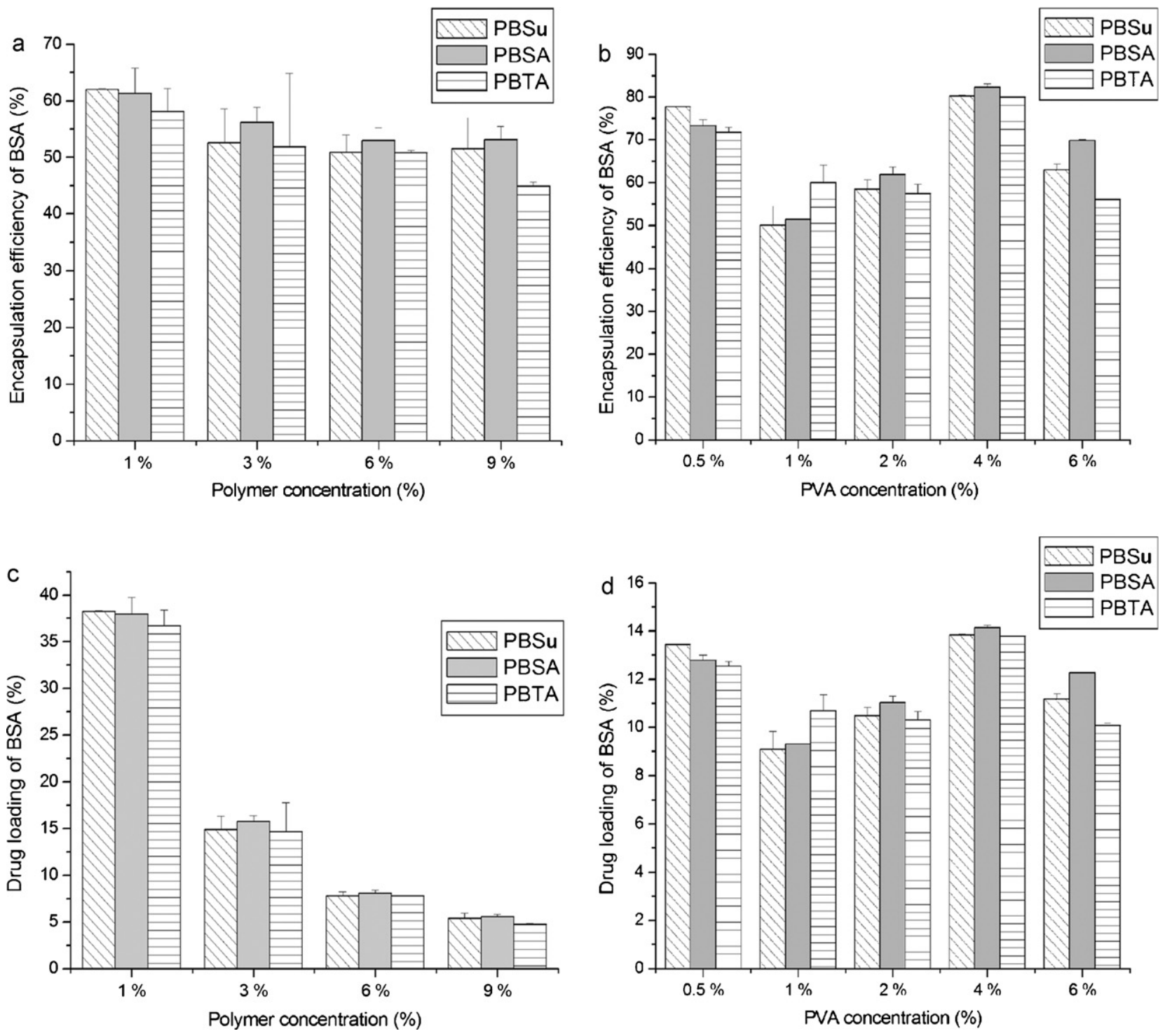

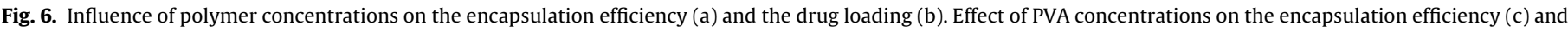
the drug loading (d). The bars represent the mean value of the data $(n=3)$ and positive standard deviation of the mean is shown.

concentration of $2 \%$ PVA, the DL values were near $11 \%$ for all three types of polyester microcapsules developed. At the highest PVA concentration, the DL values were similar to the ones obtained for 2\% PVA concentration.

The effect of PVA on the encapsulation of proteins can be quite variable. Benoit et al. reported results of the effect of the PVA concentration between 0.5 and $10 \%$ in $w_{2}$ aqueous phase on encapsulation of BSA into PCL microparticles [22]. Either lower or higher concentrations gave about $1-1.6 \%$ encapsulation efficiencies of BSA. This value increased to $4 \%$ by using an intermediate of level of PVA concentration (5\%). Also, PLGA submicron particles were shown to have EE of BSA of $90 \%$ by using an external PVA concentration in double emulsion between 0.5 and $2.5 \%(\mathrm{w} / \mathrm{v})$ [5]. A gradual decrease of EE was observed with the increase of PVA concentration above $2.5 \%$. The EE level was lower when the PVA concentration was below $0.5 \%$.

\subsection{In vitro drug release}

\subsubsection{BSA release}

A fast release of BSA was exhibited by PBSu and PBSA microcapsules in the first day. After the release of $6 \%$ of BSA from PBSu, a very slow and steady release was observed during three weeks (Fig. 7a). In the case of PBSA, after a fast release of $10 \%$ BSA, the release was continuous during 4 weeks in a linear rate and leveled off at $17 \%$. PBTA microcapsules released BSA steadily without any burst effect during one week and released a maximum of $4 \%$ of BSA. For the studied time periods, the BSA release from PBSu, PBSA and PBTA were halted nearly after 4, 3 and 1 week, respectively. The PBSA release profile shows a minor readsorption of BSA after the 4 th week. Those results indicate that the majority of the protein was encapsulated within the microcapsule interior. During the period under study, the degradation of the polyesters may not contribute significantly for the release of the encapsulated BSA.

Non-reactive functional group containing aliphatic polyester, e.g., PCL and the polyesters studied in this work, usually degrade very slowly comparing to other biodegradable polymers. The mechanism of drug release, therefore, is often dominated by the drug diffusion from the microsphere matrix, which makes such polymers suitable for very long-term release systems [42]. PCL microspheres, for example, have been shown to release BSA at a rate of approximately $30 \%$ in 1 month [43]. In our study, the microcapsules of PBTA and PBSu, containing the aqueous solution of protein, are believed to release the protein in a similar fashion than the 

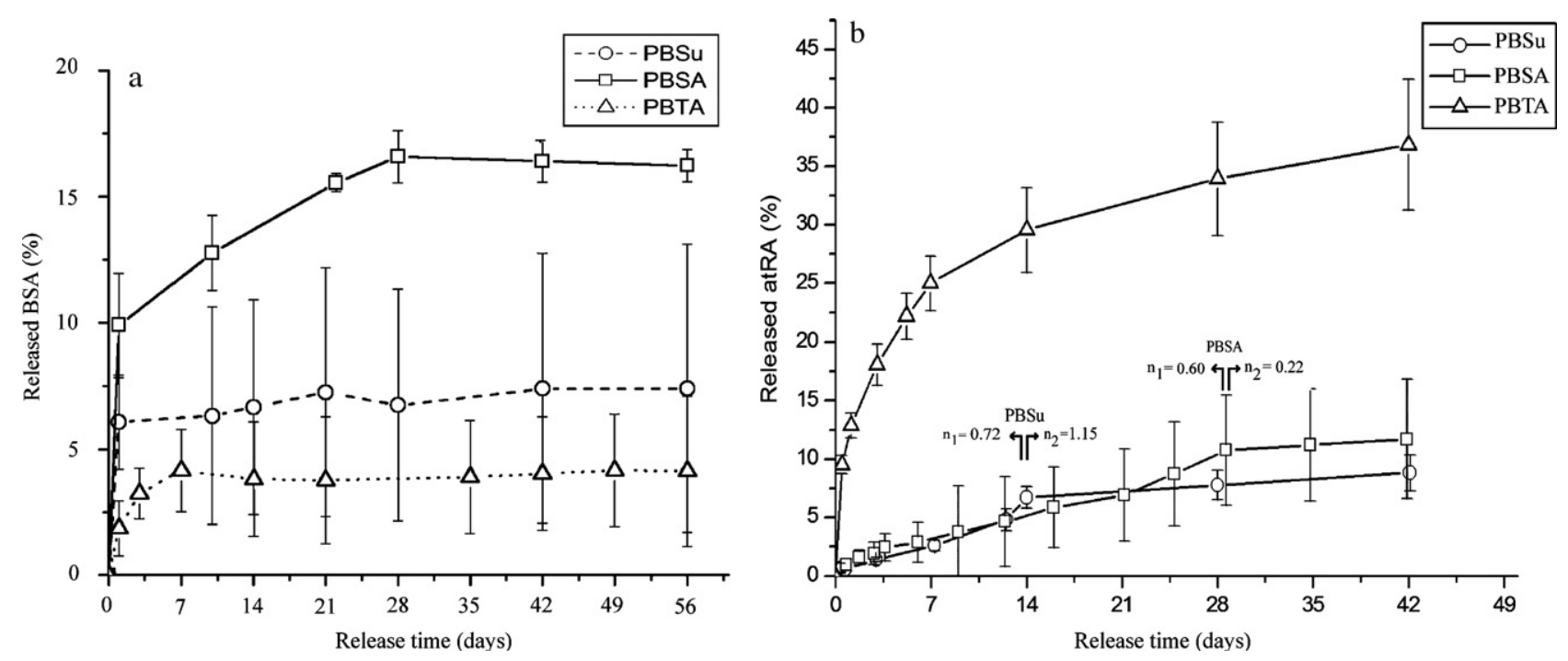

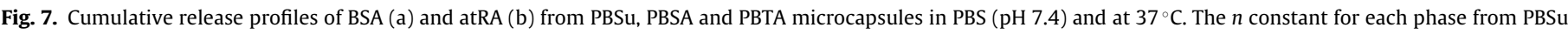
and PBSA release profile of atRA was calculated by plotting $\log$ fraction $\left(M_{t} / \mathrm{M}_{\text {inf }}\right)$ against $\log$ time.

release from a reservoir. The capsule wall acts as a barrier to prevent a fast release. The PBSA, on the other hand, showed a burst release. Although the morphology of those microcapsules is quite similar, a possible interaction of BSA with the polymer at the surface may explain the different behavior. The burst release is usually caused by the fast desorption of drugs at the surface. This mechanism is also documented in PLGA, poly(lactic acid)-poly(ethylene glycol)-poly(lactic acid), poly(ethylene oxide)-poly(lactic acid) and poly(lactic-co-hydroxymethyl glycolic acid) microspheres made by a double emulsion technique [44-47]. Very slow and steady release of BSA was observed in PBSA microcapsules until 30 days. This performance can be attributed to the controlled protein diffusion through water filled micro pores and channels. In addition, the transport of proteins in those microstructures can be governed by protein-polymer interactions, which were reported before with PLGA microspheres and its degradation products [24,48].

\subsection{2. atRA release}

The release of atRA from PBSu and PBSA microcapsules showed similar profiles and kinetic behavior. In both preparations, no burst release was observed. The release profiles show a steady rate and phases of faster and slower rates (Fig. 7b). During the first phase of two weeks, the PBSu microcapsules released atRA at a steady rate until 7\%. Afterwards, the release continued steadily at a slower rate until day 42 , with a cumulative release of about $9 \%$. For the PBSA microcapsules, except for initial 3 days where the release was fast and parabolic, the release of atRA continued linearly until the end of 24 days with a total of $10 \%$ of cumulative release. Later, in the second phase a relatively slower rate of release was detected until day 42 , with a cumulative release of $12 \%$.

The release profile of PBTA microcapsules was quite different from PBSu and PBSA, showing no burst phase and having a linear kinetic profile. A rapid burst release of atRA was, however, observed from PBTA microcapsules in the first day, releasing about $10 \%$ of drug. Then, the release until $25 \%$ has a parabolic profile that develops during the first week (Fig. 7b). During the next 5 weeks, a slower rate of release was detectable while still keeping the parabolic profile. The lack of phases and higher rate of release from PBTA microcapsules may indicate that it is less crystalline relative to PBSu and PBSA. It was reported before that PBSu has a higher degree of crystallinity (62\%) than PBSA (53\%), as determined by differential scanning calorimetry [49]. As expected from the elastic and rubbery nature of the polymer, PBTA, it shows the lower crystallinity among the studied polymers. Previously, the crystallinity of PBTA fibers prepared at high extrusion speed to increase the level of orientation and increase the crystallinity reached a value of about 30\% [50]. As the contact angle measurements showed in this study, the PBTA polyesters were relatively more hydrophilic when compared with PBSu and PBSA. This enables an easier diffusion of aqueous solutions into the microstructure of PBTA microcapsules and, consequently, may facilitate atRA transport.

The crystallinity was shown to be a major parameter in mediating the release pattern and kinetics of release from synthetic polymers. For example, lidocaine and lidobase followed three-phase and biphasic release patterns from amorphous PLGA microparticles, respectively [51]. The same drugs exhibited a single diffusion phase from semi-crystalline poly(lactic acid) (PLA) microparticles. Similarly to our results obtained with PBSu microcapsules, pseudo-zero-order release of atRA was detected for 5 weeks from PEG and PLLA diblock copolymers [41]. Conversely, amorphous microspheres of PLGA revealed a burst release pattern of atRA before a continuous or zero-order kinetics being established for longer release periods [52,53]. Also, poly(propylene-co-butylene succinate)(PPBSu) nanoparticles encapsulating hydrophobic nimodipine, performed a constant drug release without showing a burst profile [54]. In this drug delivery system, the low crystallinity was found to facilitate the release rate.

The swelling and erosion from PBSu and its copolymers can be considered negligible for the time scale of the experiments, and consequently, the diffusion will be the main mechanism of the drug release. Mathematical modelling of the release profiles using the Power law $\left(M_{t} / M_{\text {inf }}=K t^{n}\right)$ was performed to verify this hypothesis [55]. $M_{t}$ and $M_{\text {inf }}$ are the absolute cumulative amount of drug released at time $t$ and infinite time, respectively; $k$ is a constant incorporating structural and geometric characteristic of the device, and $n$ is the release exponent, indicative of the mechanism of drug release. For thin films and spheres, $n$ values of 0.5 and 0.43 are considered indicative of a Fickian release profile. For the same morphology, values of $n$ of 1 and 0.85 , respectively, are considered indicative of a zero-order release. Intermediate values are considered a mix of the two mechanisms. The studied microcapsules of PBSu, PBSA and PBTA had spherical shapes with a thin capsular wall and hollow capsular space. Therefore, a complex behavior, intermediate to those of thin films and spheres, could be expected. The $n$ constant of atRA from PBSu were $0.72-1.15$ and the release was mixed with a near zero-order character for the first and sec- 
ond release phases, respectively (Fig. 7b). The release of atRA from PBSA resulted in $n$ values of 0.60 and 0.22 , and those show that the release in the first phase must be a Fickian type of transport while the second phase must be affected and controlled by the matrix properties at the microcapsule wall.

The different forms of carriers from biodegradable and synthetic polymers which were used for encapsulating atRA exhibited various release profiles. Recently, it was reported that the PLGA films could present a sustained release of atRA for 5 weeks with a zeroorder profile [56]. The PLGA electrospun-fibrous meshes performed a sustained release of retinoic acid for 14 weeks with a parabolic shape of release profile [57]. Also, a faster release of atRA was reported from the electrospun-cellulose acetate fiber-mats with a release rate of $100 \%$ within $6 \mathrm{~h}$ when the Twen $80^{\circledR}$ was used as an emulsifier in the medium [58]. In a recent study, it was also shown that, after a burst release within the first day, the nanospheres of PCL and PLA could release $90 \%$ of atRA steadily in $240 \mathrm{~h}$ [59].

Slow atRA release rates over prolonged periods were observed from microcapsules of PBSu, PBSA and PBTA. The low dosage delivery of atRA can be necessary for a safe delivery, since high doses may be toxic. Subcutaneously administrated atRA loaded PDLLA microspheres showed a plasma level range of $3.5-25 \mathrm{ng} / \mathrm{ml}$ for $2-3$ weeks, which induced severe toxicity in rats when the plasma level exceeded $10 \mathrm{ng} / \mathrm{ml}$ [60]. In this respect, the microcapsules developed in this study can effectively deliver atRA in a safe, predictable and prolonged release rate for optimum drug bioactivity.

\section{Conclusions}

The microcapsules of biodegradable and biocompatible PBSu, PBSA and PBTA polyesters were prepared by double emulsion/solvent evaporation for encapsulation of hydrophobic and hydrophilic drugs. Smooth and spherical microcapsules of PBSA and PBTA were obtained with 1 and 3\% polymer concentrations. Multicompartment and polygonal shape microcapsules with rougher surfaces were observed between 6 and $9 \%$ polymer concentrations. The average size of the microcapsules was increased significantly between 1 and 6\% polymer concentrations and there was a sharp increase of its size at $9 \%$. The increase of PVA concentration in the second water phase decreased the size of all type of microcapsules and the capsule walls were detected to be smoother and more spherical at $2 \%$ or higher PVA concentrations. Although the variation of polymer or PVA concentration affected DL and EE of BSA significantly, there was a very small difference between the microcapsules obtained with the three polyesters at a particular concentration. Independently of the polymer type, the optimum EE was found as $60 \%$ with $1 \%$ polymer concentration. The highest values of BSA encapsulation efficiency were 75 and $70 \%$ using 0.5 and $4 \%$ PVA concentration, respectively. Protein release rate was highest and longest from PBSA microcapsules as it showed $10 \%$ of release in the first day and continued during four weeks until reaching a maximum value of $17 \%$. The atRA release from all microcapsules was continuous for prolonged periods. The release of atRA from PBSu and PBSA microcapsules indicated a near zero-order release followed by a Fickian release during six weeks. The release from PBTA microcapsules was four times faster and having a parabolic shape caused by the more hydrophilic and amorphous nature of this polymer. The results showed that microcapsules were successfully produced having release profiles relevant for a long and sustained delivery of hydrophilic and hydrophobic drugs.

\section{Acknowledgements}

This work was supported by INTERREG III A project PROTEUS - conversion of natural marine resources and residues into high added value products for industrial application.

\section{References}

[1] P.C. Besssa, M. Casal, R.L. Reis, Tissue Eng. Regen. Med. 2 (2008) 81.

[2] V. Luginbuehl, L. Meinel, H.P. Merkle, B. Gander, J. Pharm. Biopharm. 58 (2004) 197

[3] M. Biondi, F. Ungaro, F. Quaglia, P.A. Netti, Adv. Drug Deliv. Rev. 60 (2008) 229.

[4] G.A. Silva, O.P. Coutinho, P. Ducheyne, R.L. Reis, J. Tissue Eng. Regen. Med. (2007) 97.

[5] T. Feczkó, J. Tóth, J. Gyenis, Colloids Surf. A 319 (2008) 188.

[6] V.R. Sinha, A. Trehan, J. Control. Release 90 (2003) 261.

[7] D.Y. Arifin, L.Y. Lee, C. Wang, Adv. Drug Deliv. Rev. 58 (2006) 1274.

[8] V. Lemaire, J. Bélair, P. Hildgen, Int. J. Pharm. 258 (2003) 95.

[9] S. Mao, J. Xu, C. Cai, O. Germershaus, A. Schaper, T. Kissel, Int. J. Pharm. 334 (2007) 137.

[10] C. Zhu, Z. Zhang, Q. Liu, Z. Wang, J. Jin, J. Appl. Polym. Sci. 90 (2003) 982.

[11] M.L. Alves da Silva, A. Crawford, J.M. Mundy, V.M. Correlo, P.C. Sol, P.V. Hatton, R.L. Reis, N.M. Neves, Tissue Eng. Part A 13 (2007) 1735

[12] D.F. Coutinho, I.H. Pashkuleva, C.M. Alves, A.P. Marques, N.M. Neves, R.L. Reis, Biomacromolecules 9 (2008) 1139.

[13] A.R. Costa-Pinto, A. Salgado, V.M. Correlo, P. Sol, M. Bhattacharya, P. Charboard, R.L. Reis, N.M. Neves, Tissue Eng. Part A 14 (2008) 1049.

[14] J.T. Oliveira, V.M. Correlo, P.C. Sol, A.R. Costa Pinto, P.B. Malafaya, A.J. Salgado, M. Bhattacharya, P. Charbord, N.M. Neves, R.L. Reis, Tissue Eng. Part A 14 (2008) 1651.

[15] J.L. Napoli, Clin. Immunol. Immunopathol. 80 (2002) 52

[16] E.T. Baran, N. Özer, V. Hasırcı, J. Microencapsul. 19 (2002) 363.

[17] M.M. Bradford, Anal. Biochem. 72 (1976) 248

[18] S.S. D’Souza, P.P. DeLuca, Pharm. Res. 23 (2006) 460.

[19] O.H. Lowry, N.J. Rosbrough, A.L. Farr, R.J. Randall, J. Biol. Chem. 193 (1951) 265

[20] Y. Choi, S.Y. Kim, K. Park, J. Yang, K.-J. Cho, H.J. Kwon, Y. Byun, Int. J. Pharm. 320 (2006) 45.

[21] A. Lamprecht, N. Ubrich, P.M. Hombreiro, C.M. Lehr, M. Hoffman, P. Maincent Int. J. Pharm. 196 (2000) 177.

[22] M.A. Benoit, B. Baras, J. Gillard, Int. J. Pharm. 184 (1999) 73

[23] M. Li, O. Rouaud, D. Poncelet, Int. J. Pharm. 363 (2008) 26.

[24] D. Blanco, M.J. Alonso, Eur. J. Pharm. Biopharm. 45 (1998) 285

[25] F. Salaün, E. Devaux, S. Bourbigot, P. Rumeau, Chem. Eng. J. 155 (2009) 457.

[26] T.K. Kim, J.J. Yoon, D.S. Lee, T.G. Park, Biomaterials 27 (2006) 152

[27] M. Matsumura, T. Yamamoto, P.C. Wang, K. Shinabe, K. Yasuda, Water Res. 31 (1997) 1027

[28] P. Eiselt, J. Yeh, R.K. Latvala, L.D. Shea, D.J. Mooney, Biomaterials 21 (2000) 1921.

[29] K.-L. Eckert, M. Mathey, J. Mayer, F.R. Homberger, P.E. Thomann, P. Groscurth, E. Wintermantel, Biomaterials 21 (2000) 63.

[30] L. Guangyuan, Z. Lin, K. Lijun, Z. Ling, G. Yandao, Z. Nanming, Z. Xiufang Tsinghua Sci. Technol. 11 (2006) 427.

[31] I.D. Rosca, F.W.M. Uo, J. Control. Release 99 (2004) 271

[32] S. Park, Y. Lee, S. Hong, Colloid Surf. B 47 (2006) 211.

[33] P.D. Scholes, A.G.A. Coombes, L. Illum, S.S. Davis, M. Vert, M.C. Davies, J. Control. Release 25 (1993) 145

[34] D. Lemoine, V. Preat, J. Control. Release 54 (1998) 15.

[35] M.J. Heslinga, E.M. Mastria, O. Eniola-Adefeso, J. Control. Release 138 (2009) 235.

[36] Y.-L. Zhao, F. Tian, C.-J. Liu, F. Li, N. Xing, J. Appl. Polym. Sci. 110 (2008) 3826.

[37] M.H. Perez, C. Zinutti, A. Lamprecht, N. Ubrich, A. Astier, M. Hoffman, R. Bodmeier, P. Maincent, J. Control. Release 65 (2000) 429.

[38] S.J. Lim, C.K. Kim, Int. J. Pharm. 243 (2002) 135

[39] Y.I. Jeong, M.K. Kang, H.S. Sun, S.S. Kang, H.W. Kim, K.S. Moon, K.J. Lee, S.H. Kim, S. Jung, Int. J. Pharm. 273 (2004) 95.

[40] E.Z. Szuts, F.I. Harosi, Arch. Biochem. Biophys. 287 (1991) 297

[41] Y. Choi, S.Y. Kim, K.S. Lee, C. Kim, Y. Byun, Int. J. Pharm. 215 (2001) 67.

[42] V.R. Sinha, K. Bansal, R. Kaushik, R. Kumria, A. Trehan, Int. J. Pharm. 278 (2004) 1.

[43] A. Luciani, V. Coccoli, S. Orsi, L. Ambrosio, P.A. Netti, Biomaterials 29 (2008) 4800.

[44] M. Igartua, R.M. Hernández, A. Esquisabel, A.R. Gascón, M.B. Calvo, J.L. Pedraz, Int. J. Pharm. 169 (1998) 45.

[45] P. Bouillot, N. Ubrich, F. Sommer, T. Duc, M.J. Loeffler, E. Dellacherie, Int. J. Pharm. 181 (1999) 159.

[46] A. Giteau, M.C. Venier-Julienne, A. Aubert-Pouëssel, J.P. Benoit, Int. J. Pharm. 350 (2008) 14

[47] A.H. Ghassemi, M.J. van Steenbergen, H. Talsma, C.F. van Nostrum, W. Jiskoot D.J.A. Crommelin, W.E. Hennink, J. Control. Release 138 (2009) 57.

[48] A. Schoubben, P. Blasi, S. Giovagnoli, L. Perioli, C. Rossi, M. Ricci, Eur. J. Pharm. Sci. 36 (2009) 226.

[49] B.D. Ahn, S.H. Kim, Y.H. Kim, J.S. Yang, J. Appl. Polym. Sci. 82 (2001) 2808

[50] X.Q. Shi, H. Ito, T. Kikutani, Polymer 46 (2005) 11442

[51] A. Frank, S.K. Rath, S.S. Venkatraman, J. Control. Release 102 (2005) 333.

[52] Y.I. Jeong, M.K. Kang, H.S. Sun, S.S. Kang, H.W. Kim, K.S. Moon, K.J. Lee, I.S. Kim, S.H. Kim, Int. J. Pharm. 257 (2003) 195

[53] Y. Çırpanlı, N. Ünlü, S. Çalış, A.A. Hıncal, J. Microencapsul. 22 (2005) 877

[54] S. Papadimitriou, G.Z. Papageorgiou, F.I. Kanaze, M. Georgarakis, D.N. Bikiaris, J. Nanomater. (2009), doi:10.1155/2009/716242.

[55] J. Siepmann, N.A. Peppas, Adv. Drug Deliv. Rev. 48 (2001) 139.

[56] M.J. Lee, S.E. Jin, C.K. Kim, H.K. Choung, H.J. Kim, J.M. Hwang, Am. J. Ophthalmol. 148 (2009) 566 . 
[57] D. Puppi, A.M. Piras, N. Detta, D. Dinucci, F. Chiellini, Acta Biomater. 6 (2010) 1258.

[58] P. Taepaiboon, U. Rungsardthong, P. Supaphol, Eur. J. Pharm. Biopharm. 67 (2007) 387.
[59] T. Hammady, A. El-Gindy, E. Lejmi, R.S. Dhanikula, P. Moreau, P. Hildgen, Int. J. Pharm. 369 (2009) 185.

[60] Y. Choi, C. Lee, K. Park, S.Y. Kim, S.H. Kim, S. Han, S.H. Kim, Y. Byun, Drug Dev. Res. 59 (2003) 326 